December 2005

\title{
Gas Flow Across Gaps in Protoplanetary Disks ${ }^{1}$
}

\author{
STEVe H. Lubow \\ Space Telescope Science Institute, 3700 San Martin Drive, Baltimore, MD 21218, USA \\ lubow@stsci.edu \\ AND \\ Gennaro D'Angelo \\ School of Physics, University of Exeter, Stocker Road, Exeter EX4 4QL, United Kingdom \\ gennaro@astro.ex.ac.uk
}

\begin{abstract}
We analyze the gas accretion flow through a planet-produced gap in a protoplanetary disk. We adopt the alpha disk model and ignore effects of planetary migration. We develop a semi-analytic, one-dimensional model that accounts for the effects of the planet as a mass sink and also carry out two-dimensional hydrodynamical simulations of a planet embedded in a disk. The predictions of the mass flow rate through the gap based on the semi-analytic model generally agree with the hydrodynamical simulations at the $25 \%$ level. Through these models, we are able to explore steady state disk structures and over large spatial ranges. The presence of an accreting $\sim 1 M_{J}$ planet significantly lowers the density of the disk within a region of several times the planet's orbital radius. The mass flow rate across the gap (and onto the central star) is typically $10 \%$ to $25 \%$ of the mass accretion rate outside the orbit of the planet, for planet-to-star mass ratios that range from $5 \times 10^{-5}$ to $1 \times 10^{-3}$.
\end{abstract}

Subject headings: accretion, accretion discs — hydrodynamics — planets and satellites: general

\section{Introduction}

The presence of $\mathrm{a} \sim 1 M_{J}$ planet influences the structure of a circumstellar disk. The most obvious effect is the opening of a tidally produced gap (Lin \& Papaloizou 1993). Recent studies of young stars such as CoKu Tau/4 (D'Alessio et al. 2005), TW Hya (Calvet et al. 2002), KH 15D (Herbst et al. 2002), HR 4796A (Schneider 1999), and HD 141569A (Clampin et al. 2003) suggest the presence of inner holes in circumstellar disks. One possible mechanism for producing such a hole is the tidal barrier created by a planet. A planet's tidal forces can, in principle, prevent material from accreting across the orbit of the planet. The unreplenished material interior to the planet's orbit accretes onto the central star, thereby producing a hole. However, alternative explanations for apparent holes have been offered for some cases, such as dust segregation (Takeuchi \& Artymowicz 2001).

This paper deals with the determination of the extent to which the disk material exterior to a planet's orbit can replenish the interior material. A planet of $\sim 1 M_{J}$ opens a gap in a disk having typical parameters. But the gap is not clean and is accompanied by accretion onto the planet and

\footnotetext{
${ }^{1}$ To appear in The Astrophysical Journal sometime in 2006.
}

accretion flow across the gap (Artymowicz \& Lubow 1996; Bryden et al. 1999; Kley 1999; Lubow, Seibert, \& Artymowicz 1999). Several other past studies of planet-disk interactions have concentrated on analyzing torques and accretion flows onto planets. They also have sometimes reported finding mass flow through gaps (e.g., Winters, Balbus, \& Hawley 2003), while other studies have reported little inflow (e.g., Bate et al. 2003).

The extent of the flow through the gap depends on the planet mass and disk properties. In the study of a $1 M_{J}$ planet that orbits a $1 M_{\odot}$ star by Lubow, Seibert, \& Artymowicz (1999), very little mass was found to accrete interior to the planet's orbit, not surprisingly, with nonaccreting boundary conditions at the inner boundary located at 0.3 times the orbital radius of the planet. On the other hand, if the interior disk was initially strongly depleted of material, then there was a substantial accretion flow that built up the interior disk. In this configuration, the accretion rate into the interior disk is comparable to the rate onto the planet. A similar density profile was considered recently by Quillen et al. (2004) to model $\mathrm{CoKu} \mathrm{Tau} / 4$. Consequently, the accretion rate onto the central star depends on the density distribution and the inner boundary conditions.

Even low mass planets which do not open gaps can affect 
Gas Flow Across Gaps in Protoplanetary Disks

the global structure of a circumstellar disk. The previous studies of flow across a gap are largely based on multidimensional hydrodynamical simulations. They are limited by their dynamic range in space, which typically involve a region that ranges from 0.4 to 6 times the orbit radius of the planet. A smaller inner boundary increases the computational overhead, due the need to take shorter timesteps because of the higher rotational velocity. Simulations are limited in time to at most a few thousand orbits of the planet, which is less than the viscous evolution timescale of a typical protostellar disk. To overcome these limitations and provide some physical insight, we develop in Section 2 a semi-analytic model for the steady state flow across the gap. This model depends on a key parameter, the accretion efficiency, which we determine by two-dimensional simulations described in Section 3. The semi-analytic model predicts the rate of accretion past the planet and the steady state density profile. The predictions are compared with results of numerical simulations. Section 4 contains the conclusions.

\section{Semi-analytic Model}

We develop a one-dimensional, semi-analytic model for a circumstellar disk containing a planet. We assume that the planet lies on a fixed circular orbit and neglect effects of migration. In this model, we separate the gap region from the main disk region. We assume that the tidal torques on the disk exerted by the planet are confined to the gap region. In reality, waves generated by the planet propagate into the main disk and exert additional torques, which we ignore. But, shocks and other processes limit the extent of wave propagation (Bate et al. 2002).

We adopt the alpha disk model for the disk turbulence throughout. If the disk turbulence is due to magnetic fields, then the presence of a planet can affect the nature of the turbulence (Nelson \& Papaloizou 2003; Winters, Balbus, \& Hawley 2003). We do not account for such effects.

We model the effect of the planet on the main disk as a mass sink that lies on the planet's orbit. The mass sink description utilizes the accretion efficiency parameter

$$
\mathcal{E}=\frac{\dot{M}_{p}}{3 \pi \nu_{p} \Sigma_{p}}
$$

where $\dot{M}_{p}$ is the accretion rate onto the planet and $\nu_{p}$ is the turbulent kinematic viscosity. Density $\Sigma_{p}$ is the disk density at the location of the planet, based on a smooth continuation (interpolation) of the density profile outside the gap to the location of the planet (Lubow, Seibert, \& Artymowicz 1999). For a narrow gap, this density is the density just outside the gap. The denominator is recognized as the standard from for the steady state rate of accretion through a viscous disk, far from the disk inner boundary (Lynden-Bell \& Pringle 1974). Since the accretion rates scale with $\nu$, we expect and find that $\mathcal{E}$ is fairly independent of $\nu$ (and $\Sigma_{p}$ ) for fixed $H / r$, as is consistent with the results of Kley (1999). The efficiency depends mainly on the planet mass $M_{p}$. One worrisome result of the numerical simulations is that $\mathcal{E}$ is found to be greater than unity. One might think that this suggests the planet is accreting more mass than the disk can supply in a steady state. We show later that steady state accretion flows can and usually do have $\mathcal{E}$ greater than unity.

The density structure of a circumstellar accretion disk evolves in time from some initial state. The initial disk state of a protostellar disk is not known. Disks tend to evolve towards a steady state in which the accretion rate is independent of radius (Lynden-Bell \& Pringle 1974; Pringle 1981). The disk evolutionary timescale in the vicinity of planets that lie within a $10 \mathrm{AU}$ of the central star is shorter than the global evolutionary timescale for a $100 \mathrm{AU}$ disk, and so a steady state will be even more likely achieved on these smaller scales. Consequently, we seek steady state solutions for the disk structure.

\subsection{One-Dimensional Equations}

With the above model, the one-dimensional equations of mass conservation and azimuthal force balance for a disk with radial velocity $u$, angular velocity $\Omega$, and surface density $\Sigma$ in a steady state $(\partial \Sigma / \partial t=0, \partial u / \partial t=0, \partial v / \partial t=0)$ Keplerian disk are

$$
\frac{1}{r} \frac{d(r \Sigma u)}{d r}=-\frac{3 \nu \Sigma \mathcal{E}}{2 r} \delta\left(r-r_{p}\right)
$$

and

$$
r^{2} \Omega \Sigma u=-\frac{d\left(3 \mu \Omega r^{2}\right)}{d r}+2 r \Sigma \Lambda(r),
$$

where $\mu=\nu \Sigma$ with kinematic turbulent viscosity $\nu$, and $\delta$ is the Dirac delta function. $\Lambda(r)$ is the torque density per unit mass produced by the tidal field of the planet

$$
\Lambda(r)=\operatorname{Sign}\left(r-r_{p}\right) \frac{f q^{2} G M_{*}}{2 r}\left(\frac{r}{\Delta_{p}}\right)^{4}
$$

where $f$ is a constant of order unity and $\Delta_{p}$ is the maximum of $H$ and $\left|r-r_{p}\right|$. These equations are identical to those in Lin \& Papaloizou (1986), except that we have added to sink term in the equation of continuity (2).

The determination of the accretion efficiency $\mathcal{E}$ in equation (2) requires a model for the capture of gas by the planet. We shall rely on two-dimensional simulations to determine its value. We use the one-dimensional model to provide initial conditions for the two-dimensional simulations. To compose the one-dimensional model, we divide space into two regions: the gap region and the main disk (nongap) region. We combine the solutions in the two regions of space to obtain approximate steady state global solutions. In the process, we self-consistently determine the accretion efficiency, which links the solutions in these two regions.

\subsection{Gap Region}

From previous two and three-dimensional studies discussed in the Introduction, we know that the gap region is complicated by the presence of nonKeplerian flow and shocks. We do not expect that a one-dimensional model would work well in describing the detailed gas flow there. But, we estimate the density structure $\Sigma_{g}$ in the gap region by assuming $d / d r \gg$ 
$1 / r$ (WKB approximation) and $1 / \Delta_{p} \gg 1 / r$ in equation (3) to obtain

$$
\Sigma_{g}(r)=\Sigma_{p} \exp \left[-\frac{f}{9} \frac{q^{2} r_{p}^{2} \Omega_{p}}{\nu_{p}}\left(\frac{r_{p}}{\Delta_{p}}\right)^{3}\right],
$$

where $\Sigma_{p}$ is the density that would occur at $r=r_{p}$ in the absence of a gap, as defined in equation (1). We generally take constant $f$ equal to 2 . We have used initial density profiles like this in our previous work because they provide good initial conditions for planet-disk simulations. They undergo moderate changes and appear to reach a dynamically steady state in the gap after a few hundred planetary orbits. The achievement of a dynamically steady state is expedited through the development of shocks.

On longer, viscous timescales, the gap region could undergo further structural changes. They arise in part because of the interaction of the gap region with the main disk region. The coupling between these two regions occurs through the accretion efficiency parameter $\mathcal{E}$, which is determined in the gap region by the outcome of the simulations. For a consistent solution, we require that the value of the accretion efficiency parameter match in the two regions, as described in the next subsection.

\subsection{Main Disk Region}

Outside the gap region, we ignore the presence of waves which we assume damp most of their energy near the planet and neglect the planetary torque $\Lambda$. The planetary accretion affects the large scale accretion flow as a mass sink. We expect that main disk region is described well by the one-dimensional model.

From equation (2), we have that

$$
\begin{array}{r}
\dot{M}_{i}=-2 \pi r u_{i} \Sigma_{i} \\
\dot{M}_{e}=-2 \pi r u_{e} \Sigma_{e} \\
\dot{M}_{e}=\dot{M}_{i}+3 \pi \nu_{p} \Sigma_{p} \mathcal{E},
\end{array}
$$

where $\dot{M}_{i}$ and $\dot{M}_{e}$ denote the mass accretion rate respectively interior and exterior to the orbit of the planet, and $\nu_{p}$ and $\Sigma_{p}$ are as used in equation (1).

We solve equation (3) subject to the zero-stress boundary condition at the inner edge to account for the effects of a boundary layer, where the disk meets the central star, as described by Lynden-Bell \& Pringle (1974). To model this effect, one typically applies a zero density boundary condition at the inner edge (e.g., Pringle, Verbunt \& Wade 1986), so that $\mu\left(r_{*}\right)=0$, where $r_{*}$ is the location of the stellar radius. The solution is given by

$$
3 \pi \mu_{i}(x)=\dot{M}_{i}\left(1-\frac{x}{x_{*}}\right)
$$

and

$$
3 \pi \mu_{e}(x)=\dot{M}_{o}+\frac{C}{x},
$$

where $x=\sqrt{r}, \mu_{p}=\mu\left(x_{p}\right)$, and $\mu_{i}\left(\mu_{e}\right)$ is the value of $\mu$ interior (exterior) to the orbit of the planet, and $C$ is a constant of integration.
For a narrow gap, it follows that

$$
\mu_{i}\left(x_{p}\right)=\mu_{e}\left(x_{p}\right)
$$

since $\mu_{i}\left(x_{p}\right)$ and $\mu_{e}\left(x_{p}\right)$ are nearly equal to the $\mu$-values on both sides of the planet just outside the gap. We combine equations (8), (9), and (10) to obtain

$$
\mu_{i}(x)=\mu_{p} \frac{x_{p}\left(x-x_{*}\right)}{x\left(x_{p}-x_{*}\right)}
$$

and

$$
\mu_{e}(x)=\mu_{p}\left[1+\left(1-\frac{x_{p}}{x}\right)\left(\mathcal{E}+\frac{x_{*}}{x_{p}-x_{*}}\right)\right],
$$

where $\mu_{p}$ is the value of $\mu$ smoothly extended from the main disk to the planet, excluding the gap, $\mu_{p}=\mu_{i}\left(x_{p}\right)=\mu_{e}\left(x_{p}\right)$.

Notice that for $\mathcal{E}=0$ (no planet) and $x_{*}=0$, the solution implies that $\mu(x)$ is constant, which is equivalent to the condition that $\dot{M}=3 \pi \nu \Sigma$ is independent of $r$ in a steady state, as discussed above.

Far outside the orbit of the planet at some $x_{o} \gg x_{p}$, the disk is hardly aware of the planet. We have from equations (3) and (13) that

$$
\dot{M}_{e}=3 \pi \mu_{o}=3 \pi \mu_{p}\left(\mathcal{E}+\frac{x_{p}}{x_{p}-x_{*}}\right) .
$$

Exterior accretion rate $\dot{M}_{e}$ is independent of $x$ for all $x>$ $x_{p}$. The accretion rate onto the planet is given by

$$
\dot{M}_{p}=3 \pi \mu_{p} \mathcal{E}
$$

and the accretion rate interior to the planet's orbit (and onto the star) is then

$$
\dot{M}_{i}=\dot{M}_{e}-\dot{M}_{p}=\frac{3 \pi \mu_{p} x_{p}}{x_{p}-x_{*}},
$$

which is constant for $x<x_{p}$.

The mass flow rate ratio of accretion past the planet to accretion onto the planet is then

$$
\frac{\dot{M}_{i}}{\dot{M}_{p}}=\frac{1}{\left(1-\sqrt{r_{*} / r_{p}}\right) \mathcal{E}} .
$$

The ratio of the accretion rate interior to the orbit of the planet to the rate exterior is given by

$$
\frac{\dot{M}_{i}}{\dot{M}_{e}}=\frac{1}{1+\left(1-\sqrt{r_{*} / r_{p}}\right) \mathcal{E}} .
$$

Ratios (17) and (18) are independent of $\mu_{p}$.

The star and planet compete for accretion flow. The above accretion rate ratios indicate that the closer the stellar surface comes to the planet, the more the star accretes and the less the planet accretes. This prediction is confirmed in the numerical simulations discussed later (model $\mathrm{b}$ versus model $\mathrm{g})$. This result is a consequence of the influence of the star in diverting flow from the planet. For a given kinematic viscosity $\nu(r)$ and accretion efficiency $\mathcal{E}$, we can determine the 
Gas Flow Across Gaps in Protoplanetary Disks

disk density profile outside the gap $\Sigma(r)=\mu(r) / \nu(r)$ from equations (12) and (13). The accretion efficiency depends on the flow details within the gap, which we determine in Section 3 based on two-dimensional hydrodynamical simulations. Equation (17) determines the ratio of the accretion rate onto the central star to the accretion rate onto the planet. This ratio can be compared against results of numerical simulations.

For high mass planets (typically several Jupiter masses), the analytic model breaks down because gravitational torques cannot be ignored in the main disk (outside the gap). This breakdown can be seen in equation (18). For a high planet mass, the gap is very clean and the accretion efficiency $\mathcal{E}$ is very small. In the limit of a (nonmigrating) high planet mass, the disk will evolve towards a decretion disk, rather than an accretion disk (Pringle 1981). A decretion disk has a very different description than the accretion disk description used here. In the high planet mass limit, equation (18) predicts that mass accretes uninhibitedly past the planet, which is incorrect. Instead, equation (18) applies to systems containing lower mass planets. In that case, $\mathcal{E}$ is small when the planet mass is small and equation (18) provides a proper description.

Notice that there is no problem with having $\mathcal{E}$ greater than unity in a steady state. The accretion rate of material outside the orbit of the planet, $\dot{M}_{e}$, is greater than $3 \pi \mu_{p}$. The dynamic viscosity $\mu_{p}$ and typically the surface density near the planet (and outside the gap) are reduced due to the presence of the planet, in accordance with equation (14).

\section{Numerical Hydrodynamical Simulations}

\subsection{Description of Code}

We carried out a series of numerical disk simulations in two dimensions using the code described in D'Angelo, Henning, \& Kley (2002); D'Angelo, Kley, \& Henning (2003). The code calculates the time-evolution Navier-Stokes equations for a gaseous disk that or bits a central star. The disk is subject to gravitational forces from a planet that lies on a fixed circular orbit. The code allows for high resolution near the planet by using set of nested grids. We adopt a locally isothermal equation of state with sound speed equal to the disk aspect ratio, $H / r$, times the local Keplerian velocity. We used a constant disk aspect ratio $H / r=0.05$ throughout, implying that the temperature distribution scales as the inverse of the distance from the disk axis. Unless otherwise stated, the kinematic viscosity at the radius of the planet is $\nu_{p}=10^{-5} r_{p}^{2} \Omega_{p}$, which is equivalent to Shakura \& Sunyaev parameter $\alpha=4 \times 10^{-3}$ at the location of the planet. We consider a range of planet masses, having a mass ratio with the central star of $q=5 \times 10^{-5}$ to $2 \times 10^{-3}$. Below about $q=4 \times 10^{-4}$ with a $1 M_{\odot}$ star, the size of the planet's Hill sphere is smaller than the disk thickness and three-dimensional effects can be important (Bate et al. 2003). Due to computational resource limitations, the simulations were carried out in two dimensions. In low end of the planet mass range we consider, numerical estimates of the accretion rate in two and three dimensions differ by only $30 \%$ (D'Angelo, Kley, \& Henning 2003). So we do not expect that three-dimensional effects will substantially alter our conclusions.

The grid size used for these calculations consisted of a three-level nested-grid system with the basic level having $374 \times 422$ grid zones in the radial and azimuthal direction, respectively. The first and the second subgrid level had $113 \times 103$ and $133 \times 123$ grid zones, respectively. The highest resolution achieved in the gap region around the planet was $\Delta r=3.7 \times 10^{-3} r_{p}$. The resolution in the azimuthal direction was equal to $\Delta r / r_{p}$.

The origin of the coordinate system is located on the star and the reference frame rotates about the disk axis at a rate equal to the angular velocity of the planet. The acceleration of the coordinate system origin relative to center-of-mass of the star-planet system is accounted for in the gravitational potential of the disk. As discussed in Section 3.3, we performed a convergence test on one of our models which indicates that this resolution is adequate. Self-gravity of the disk material is ignored.

The smoothing length of the planet potential was chosen to be 0.1 times the planet's Hill radius. Two and threedimensional models with that smoothing length yield similar accretion rates around a Jupiter mass planet (D'Angelo, Kley, \& Henning 2003). Some recent models show that variations of this smoothing by a factor of about 1.4 affects the accretion rate by about $1 \%$.

Accretion boundary conditions were generally applied to the planet. Such boundary conditions are currently thought to be appropriate to the range of planet masses considered in this paper because such planets are believed to undergo runaway gas accretion (e.g., Pollack et al. 1996). The accretion was simulated by removing mass within a radius of 0.2 Hill radii from the planet. We fix a removal time-scale, which is on the order of 0.1 orbital periods. Tests on how the accretion rate depends on these two parameters are given in D'Angelo, Henning, \& Kley (2002). The tests show that the accretion rate does not depend sensitively on the details of the mass removal parameter values, provided the removal prevents mass build-up near the planet. The formulation of planetary mass accretion in Lubow, Seibert, \& Artymowicz (1999) and Bate et al. (2003) is completely different from that adopted here. Nonetheless, they obtain accretion rates that are very similar to those obtained by (D'Angelo, Henning, \& Kley 2002; D'Angelo, Kley, \& Henning 2003). Consequently, the evidence all suggests that the accretion rate is the rate that a planet would accrete under runaway accretion conditions. It is not an artifact of the mass removal process.

The inner boundary was usually set at $0.4 r_{p}$, while the outer boundary was located at $6 r_{p}$. At the inner boundary, outflow boundary conditions were applied. This means that if $u_{r}<0$ at the first active zone, this value is transferred to the zones off the active grid (ghost zones). If $u_{r}>0$ at the first active zone, then $u_{r}$ of the ghost cells is set to zero. At the outer boundary, inflow and outflow of material are permitted. The outflow is implemented as in Godon (1996, 1997). During the course of the simulation, the values of all 
physical variables are free to change at the inner and outer boundaries. They are not fixed by the initial analytic model.

\subsection{Density Determination}

For a fixed choice of $\nu(r)$ and an initial guess of accretion efficiency $\mathcal{E}$, we determine the initial density profile from equations (12) and (13) with a superimposed gap profile (5). This profile is given by

$$
\Sigma(r)=\frac{\mu(r) \Sigma_{g}(r)}{\nu(r) \Sigma_{p}}
$$

where $\mu(r)$ refers to $\mu_{i}$ for $r<r_{p}$ and $\mu_{e}$ for $r>r_{p}$.

We generally set $r_{*}$ according to the location of the inner boundary. The initial gap profile is determined by an approximate balance of viscous torques with tidal torques, as described in Section 2.2. The value of $\mathcal{E}$ in the simulations is determined by the accretion rate onto the planet through equation (15). We run the simulation for typically 700-800 orbits at which point a nearly time-independent value of $\mathcal{E}$ is determined (see Figure 1). However, this value in general disagrees with the value assumed in constructing the density profile. We then guess another value of $\mathcal{E}$, construct the corresponding density profile, and rerun the simulation. We continue iterating until the assumed value of $\mathcal{E}$ used for the density profile matches the measured value from equation (15) to within $10 \%$. This process typically requires 3 or 4 iterations.

Although we find a nearly stationary value of $\mathcal{E}$ (Figure 1), the determination of a strict steady state profile in the gap from arbitrary initial conditions would require running the code beyond a local viscous timescale of order $10^{4}$ orbits, much longer than 700 orbits. However, the gap structure is also determined by nonlinear effects (shocks) that act on shorter timescales and are likely responsible for the nearstationarity of $\mathcal{E}$. Furthermore, from Figure 1 we see that $\mathcal{E}$ converges in time to the same value, independent of initial conditions. This gives us confidence that the value of $\mathcal{E}$ we determine from simulations is the steady state value. Nonetheless, we cannot prove that the value of $\mathcal{E}$ that we determine is rigorously the steady state value without integrating over longer timescales.

\subsection{Description of Results}

The results of the simulations are contained in Table 1. The default parameters consist of a kinematic viscosity $\nu=$ $10^{-5} r_{p}^{2} \Omega_{p}$ which is taken to be independent of radius, together with the other parameters described in Section 3.1. The first column in Table 1 contains the model label. The second column contains the planet mass ratio. The third column contains the variant, if any, on the default parameters. The fourth and fifth columns contain respectively the accretion efficiency $\mathcal{E}$ applied to the final iteration and the accretion efficiency $\mathcal{E}$ obtained from the final simulation. As discussed in Section 3.2, for each model we iterated on $\mathcal{E}$ until the applied and simulated $\mathcal{E}$ agreed to within $10 \%$. The sixth column contains the ratio of the accretion rate past the planet (and onto the central star) to the accretion rate onto the planet as obtained from the simulation. The seventh column contains the same quantity as the sixth, but obtained from equation (17) of the semi-analytic model. The eighth column contains a correction, described in Section 3.6, that is applied to the values in the seventh column, in order to account for the location of the inner boundary.

Model $\mathrm{b}$ is the default $q=1 \times 10^{-3}$ model. The next seven models are variants of model $b$ having a different initial density profiles (models $\mathrm{c}$ and $\mathrm{d}$ ), twice the kinematic viscosity (model e), a viscosity that increases as $r^{1 / 2}$ with the same $H / r=0.05$ (model f), a smaller inner boundary (model g), a lower temperature $H / r=0.03$ disk (model h), and a planet that does not accrete gas (model i).

A convergence test was performed on model b by using a degraded resolution throughout the computational domain. The resolution was reduced by $25 \%$, in each direction, relative to that presented in Section 3.1. Both in terms of accretion efficiency and accretion rate past the planet, the outcomes agree within $2 \%$ with those in the Table.

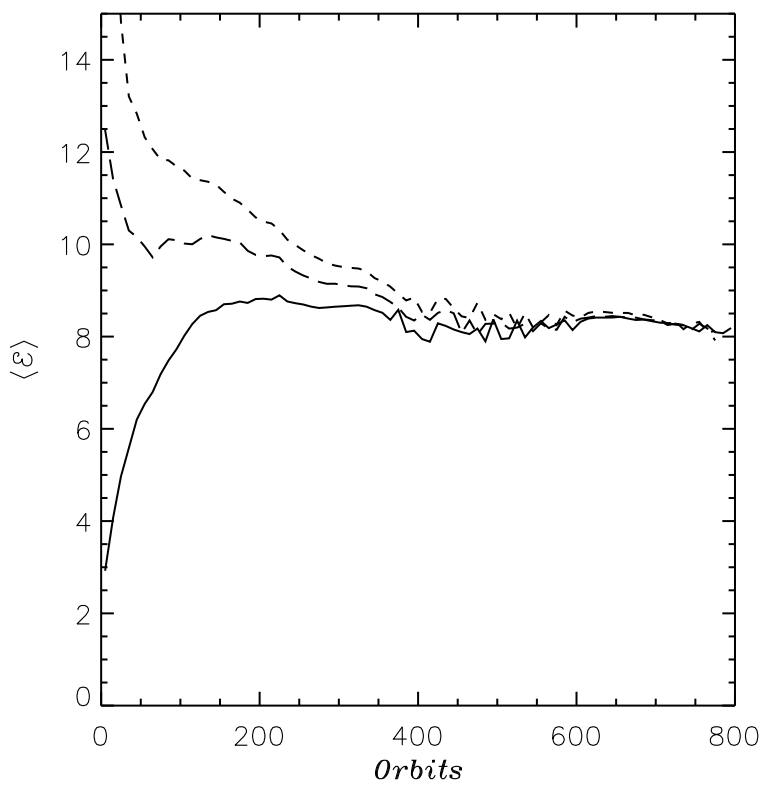

Fig. 1.- Plot of the averaged accretion efficiency $\langle\mathcal{E}\rangle$, averaged over the previous 10 planet orbits, versus time in planet orbit periods for a disk containing a $q=1 \times 10^{-3}$ planet. The models in the three cases have different initial density distributions in the gap region. The curves from lowest to highest correspond to models b, c, and d of Table 1, which have values of $f=2,1$, and 0.5 , respectively for the initial gap density distribution of equation (5). 
Gas Flow Across Gaps in Protoplanetary Disks

Table 1: Results from simulations (see Section 3.3)

\begin{tabular}{cccccccc}
\hline \hline Model & $q$ & variant & $\begin{array}{c}\text { Applied } \\
\mathcal{E}\end{array}$ & $\begin{array}{c}\text { Simulated } \\
\mathcal{E}\end{array}$ & $\begin{array}{c}\text { Simulated } \\
\dot{M}_{i} / \dot{M}_{p}\end{array}$ & $\begin{array}{c}\text { Predicted } \\
\dot{M}_{i} / \dot{M}_{p}\end{array}$ & $\begin{array}{c}\text { Corrected } \\
\dot{M}_{i} / \dot{M}_{p}\end{array}$ \\
\hline $\mathrm{a}$ & $2 \times 10^{-3}$ & & 3 & 2.9 & 0.52 & 0.91 & \\
$\mathrm{~b}$ & $1 \times 10^{-3}$ & & 8 & 8.3 & 0.38 & 0.34 & 0.13 \\
$\mathrm{c}$ & $1 \times 10^{-3}$ & $f=1$ & 8 & 8.3 & 0.38 & 0.34 & 0.13 \\
$\mathrm{~d}$ & $1 \times 10^{-3}$ & $f=0.5$ & 8 & 8.4 & 0.39 & 0.34 & 0.13 \\
$\mathrm{e}$ & $1 \times 10^{-3}$ & $2 \times \nu$ & 8 & 8.8 & 0.35 & 0.34 & 0.13 \\
$\mathrm{f}$ & $1 \times 10^{-3}$ & $\nu \propto r^{1 / 2}$ & 8 & 8.2 & 0.40 & 0.34 & 0.13 \\
$\mathrm{~g}$ & $1 \times 10^{-3}$ & $r_{i}=0.22 r_{p}$ & 9 & 9.4 & 0.14 & 0.11 & 0.11 \\
$\mathrm{~h}$ & $1 \times 10^{-3}$ & $H / r=0.03$ & 6 & 6.3 & 0.29 & 0.46 & \\
$\mathrm{i}$ & $1 \times 10^{-3}$ & $\dot{M}_{p}=0$ & 0 & 0 & & & \\
$\mathrm{j}$ & $5 \times 10^{-4}$ & & 12 & 12.4 & 0.35 & 0.23 & 0.08 \\
$\mathrm{k}$ & $1 \times 10^{-4}$ & & 7 & 7.3 & 0.50 & 0.39 & 0.14 \\
$\mathrm{l}$ & $5 \times 10^{-5}$ & & 3 & 3.2 & 1.10 & 0.91 & 0.33 \\
\hline
\end{tabular}

\subsection{Accretion Efficiency}

As can be seen from the Table, the accretion efficiency is largely a function of planet mass and is insensitive to the initial density profile (models $\mathrm{c}$ and $\mathrm{d}$ ). It is also insensitive to the viscosity and its variation in $r$, and inner boundary location which are varied in models e through $\mathrm{g}$, relative to model b. The efficiency varies from a low value of 3 for the highest and lowest mass planets considered, to a high value of 12 at an intermediate mass. This behavior is easily understood. At the high mass end, $q=2 \times 10^{-3}$ (model a), the tidal field of the planet is so strong that material is inhibited from accreting onto the planet, resulting in a lower $\mathcal{E}$ value relative to model b of $q=1 \times 10^{-3}$.

However, the accretion across the gap is somewhat less effected, since $\dot{M}_{i} / \dot{M}_{p}$ is larger for $q=2 \times 10^{-3}$ than for $1 \times 10^{-3}$. While at the low mass end (model 1 ), the planet's gravitational field has less ability to attract and accrete matter. For comparison, the Jupiter-mass models $\left(q=1 \times 10^{-3}\right)$ in D'Angelo, Kley, \& Henning (2003) and Bate et al. (2003) have an efficiency of about 4 . As discussed in the Introduction, a planet's accretion rate is influenced by the density structure outside the gap $\left(r>r_{p}\right)$, which in the present steady state case is different from that used in the above papers.

\subsection{Accretion Past Planet}

The last three columns in Table 1 describe the rate at which material flows past the orbit of the planet as a fraction of the accretion rate onto the planet. The values predicted by equation (17) generally agree with the values obtained by the simulations at the $25 \%$ level.

We believe model a $\left(q=2 \times 10^{-3}\right)$ is in the high mass regime where the semi-analytic model breaks down because gravitational torques in the main disk cannot be ignored, as discussed in Section 2.3 for decretion disks. The disk in this case is not a true decretion disk because there is some accretion through the gap. However, the accretion efficiency is markedly lower than that in model b which has $q=1 \times 10^{-3}$. We regard model a as a transitional disk between accretion and decretion. We believe this explains the discrepancy between the simulated and predicted accretion ratios.

For model b $\left(q=1 \times 10^{-3}\right)$ the simulated and predicted accretion rate ratios are in very good agreement, suggesting that the disk with such a planet behaves as an accretion disk. Agreement is also very good for model e in which the disk viscosity has been doubled and model $\mathrm{f}$, in which the $\nu$ increases with radius.

Model $\mathrm{g}$ has a smaller inner boundary located at $0.22 r_{p}$, instead of the default $0.4 r_{p}$, and used an initial density profile that has $r_{*}=0$. It attempts to provide more realistic coverage of the interior disk, but its computational domain does not cover the assumed profile extent, as do all the other models (which have $r_{*}=0.4 r_{p}$ ). Both the simulated and predicted flow rates indicate that the flow rate past the planet decreases significantly for a smaller inner boundary radius.

Model h has a colder disk and consequently the tidal effects of the planet on the disk which open the gap are relatively stronger than pressure and viscosity. This case is intermediate between models $\mathrm{a}$ and $\mathrm{b}$, with accretion efficiency lower than for model $b$. The simulated and predicted mass flow ratios in columns 6 and 7 do not agree well, probably for similar reasons given for model a. The flow onto the planet and across the gap are both reduced by about $25 \%$ relative to model b.

Model i has nonaccreting boundary conditions onto a planet having $q=1 \times 10^{-3}$. Consequently, we have applied an initial model having $\mathcal{E}=0$ to the simulation. The accretion 
rate obtained from the simulation agrees with the predicated steady state accretion rate, equation (16), to within $5 \%$. The accretion rate onto the star should be unaffected by the presence of the planet.

The agreement on flow rates for model $\mathrm{j}$ is the least satisfactory of all models having $H / r=0.05$ and $q \leq 1 \times 10^{-3}$. It is not clear why this is the case. It may have to do with the high accretion efficiency, which is also observed in threedimensional calculations (D'Angelo, Kley, \& Henning 2003). Fairly good agreement $(\sim 20 \%)$ between the simulated and predicted accretion rates is found for the lowest mass models $\mathrm{k}$ and $\mathrm{l}$.

\subsection{Correction Due To Inner Boundary}

For planets whose orbital radii are of order $\mathrm{AU}$, the inner boundary radius is relatively small, $r_{*} \lesssim 0.01 r_{p}$. It is difficult to directly simulate a disk with a small inner boundary, due to the shortness of timesteps required. We estimate a correction factor for the boundary location to be applied to the accretion rates. We saw in the previous subsection that the accretion efficiency $\mathcal{E}$ depends mainly on planet mass and is insensitive to the inner boundary location. Using the default inner boundary location of $0.4 r_{p}$ and applying equation (17), we suggest that a correction factor to the flow rate ratio for a small radius inner boundary is about $1-\sqrt{0.4} \approx 0.37$. This factor is applied to the predicted accretion rate ratios (except for models a and $\mathrm{h}$ for which the analytic model does not apply, model i which does not allow accretion onto the planet, and model $\mathrm{g}$ which already has $r_{*}=0$ ). The result is in the eighth column of Table 1.

The ratio of accretion rate onto the star to the rate onto the planet varies with mass ratio. The ratio is about $13 \%$ for a $q=1 \times 10^{-3}$ planet and $33 \%$ for a $q=5 \times 10^{-5}$ planet. From equation (18) with $r_{*}=0$, the ratio of the accretion rate onto the star to the accretion rate outside the orbit of the planet is $11 \%$ for a $q=1 \times 10^{-3}$ planet and $25 \%$ for a $q=5 \times 10^{-5}$ planet.

\subsection{Density Profiles}

We examine the effect of a planet on the density distribution of a steady state viscous disk. Figure 2 shows the assumed density profile and evolved density profile for several models which are converged in $\mathcal{E}$. For all the cases, the code uses the same boundary conditions, as discussed in Section 3.1. There are some differences in the profiles as a consequence of evolution, including some narrowing of the gap. Overall, however, the agreement between the analytic (initial) profile and the evolved one is very good.

Global density profiles with a more realistic inner boundary location $\left(r_{*}=0\right)$ were constructed for a $q=1 \times 10^{-3}$ planet using $\mathcal{E}=8$ (see Table 1 ). A global analytic density distribution is obtained from equation (19) for some assumed viscosity function $\nu(r)$. In addition, density profiles for corresponding disks without a planet were also determined. The results are shown in Figure 3. These density distributions do not contain the evolutionary effects that in principle could be determined by numerical simulations. But as can be seen from Figure 2, these effects are modest, at least over 700-800 orbits. Furthermore, it would be impossible to carry out numerical simulations with a realistically small inner boundary due to the shortness of timesteps required. The cases without planets correspond to steady disks that have the same viscosity distribution at all $r$ and the same density distribution at $r \gg r_{p}$ as the corresponding cases with planets. (For the left plot in Figure 3, the density match occurs at distances larger than shown). If an accreting planet were placed in a disk having the non-planet density distribution, indicated by dashed curves in Figure 3, then over time it would evolve towards the density distributions, indicated by the solid curves in Figure 3.

The accreting planet effects the disk density distribution over several $r_{p}$. For $r \gg r_{p}$, the fractional density difference between the nonplanet and planet cases varies slowly as $\sim$ $\sqrt{r_{p} / r}$. Consequently, the planet's effects on the disk extend over several times $r_{p}$. Also plotted are the cases with an inner boundary of $r_{*}=0.4 r_{p}$, as was generally adopted in the numerical simulations. Notice that such cases agree well with the $r_{*}=0$ cases for $r>r_{p}$, but poorly represent the density at small $r$.

\section{Discussion and Summary}

We have investigated two-dimensional steady state configurations of a protostellar disk containing a planet that undergoes mass accretion, using both an analytic model and numerical simulations. The planet is assumed to be of fixed mass and on a fixed circular orbit. Planet migration and mass gain can be neglected at late stages of evolution where the disk mass is less than the planet mass. The results also suggest the outcome for the more general case involving mass gain and migration.

We speculate on the nonsteady effects of planet mass gain and migration. The mass doubling timescale for Jupiter undergoing runaway gas accretion from the minimum mass solar nebula is of order the local viscous timescale in our model ( $\sim 10^{5}$ years). Consequently, the nonsteady effects of planet mass gain are of possible importance in that case. Consider the case of an initially steady accretion disk in which a planet of mass ratio $q \lesssim 1 \times 10^{-3}$ grows and increases its mass on a timescale shorter than the viscous timescale. We expect that the accretion rate onto the central star corresponds to the steady state rate at an earlier time when the planet mass was lower. Therefore, we expect that the accretion rate onto the star to be at least $\sim 10 \%$ of the accretion flow rate outside the planet's orbit in this mass range.

For an inwardly migrating planet, the available time to produce an inner hole is limited by the planet's migration timescale. Furthermore, a planet that moves with the accretion flow (Type II migration) would not be expected to have much effect on the accretion rate onto the central star. Current migration models in the planet mass range considered here $\left(0.05\right.$ to $\left.1 M_{J}\right)$, suggest that migration occurs on about the local viscous timescale (e.g., Bate et al. 2003). We then 

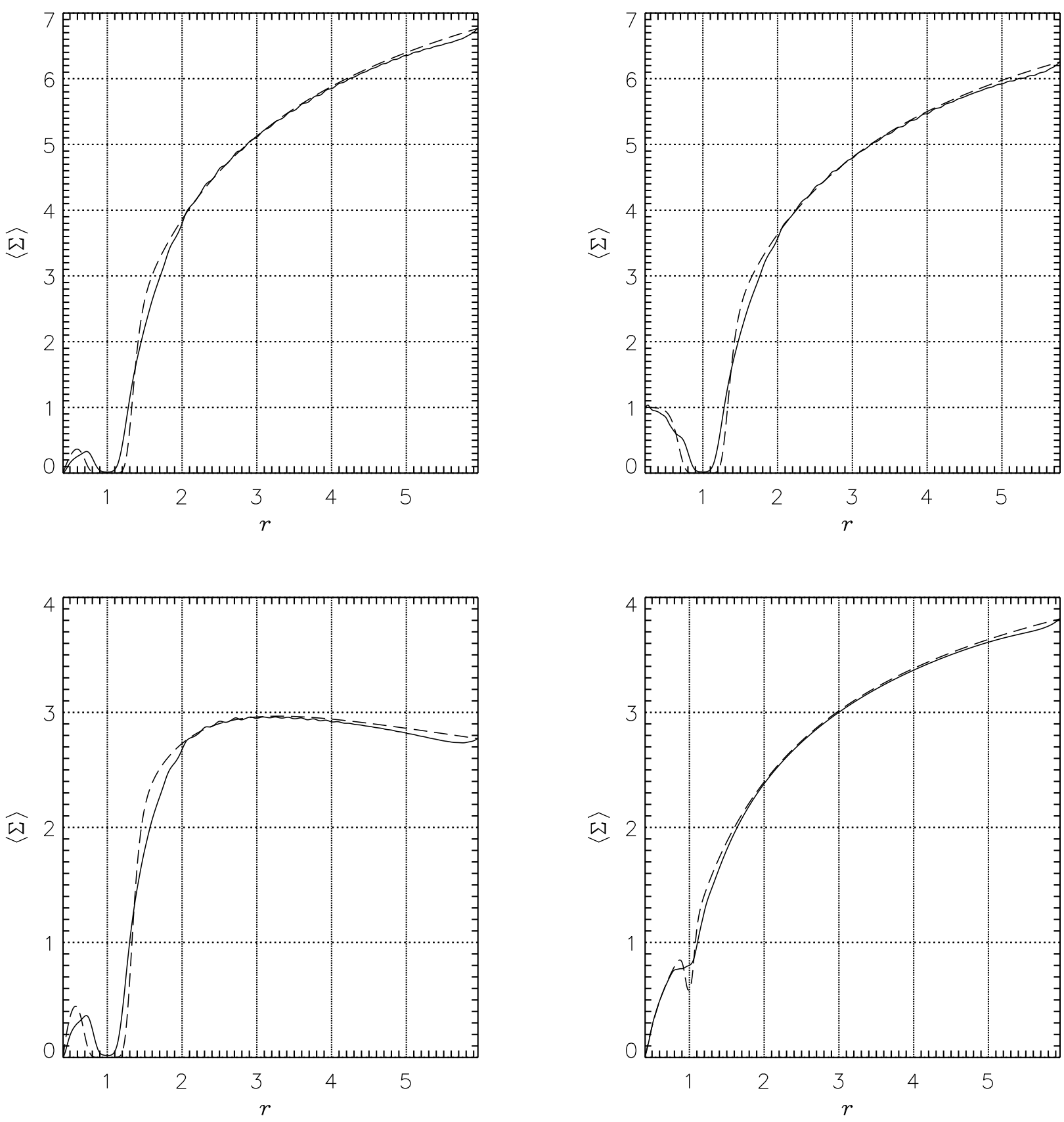

Fig. 2.- Plots of azimuthally-averaged density in units of $\Sigma_{p}$ versus radius in units of $r_{p}$ for the unevolved (dashed) and evolved (solid) models b (top-left), g (top-right), f (bottom-left), and l (bottom-right) in Table 1.

expect that an inwardly migrating planet at some orbit radius would have a more substantial interior disk and higher accretion rate onto the star than a planet on a fixed orbit at the same radius. Both the effects of planet mass gain and migration appear to lead to less depleted inner disks than the steady state models suggest.
Present-day multi-dimensional hydrodynamical simulations are incapable of following a typical protostellar disk to a steady state with spatial coverage down to the central star. To circumvent this problem, we have developed a one-parameter (namely the accretion efficiency) family of semi-analytic steady state solutions for the main disk, ex- 

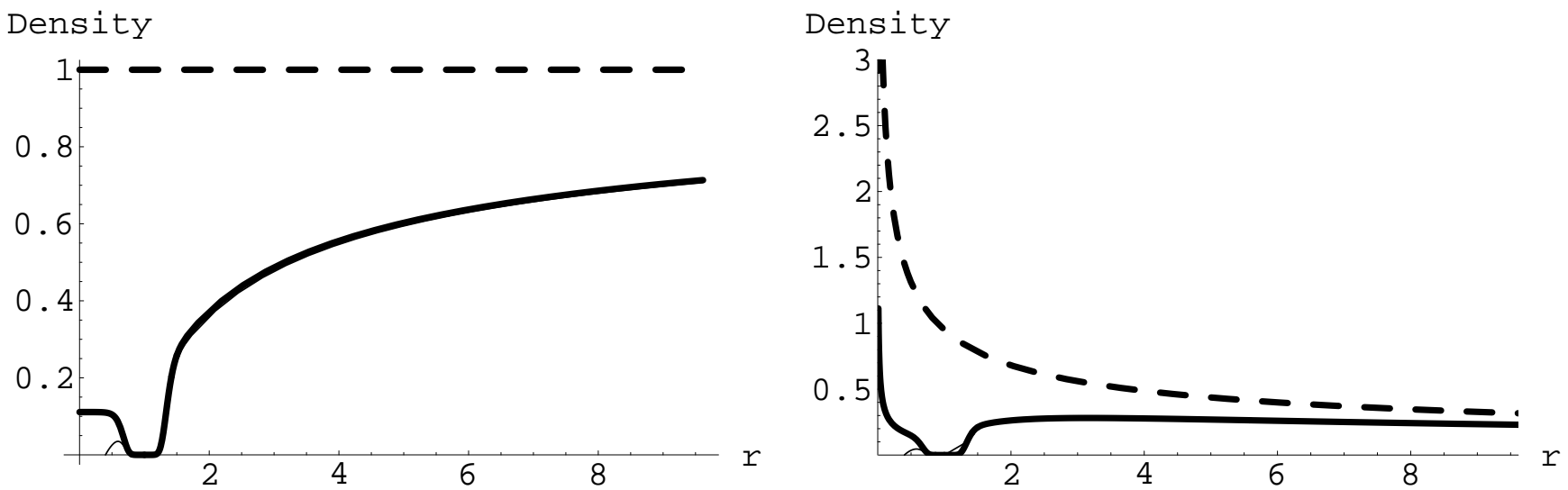

Fig. 3.- Density (in units described below) for a steady state disk containing an accreting planet, whose mass ratio is $q=1 \times 10^{-3}$, is plotted as a function of radius in units of $r_{p}$. The left plot (model b of Table 1 ) is for a constant disk viscosity and the right plot is for a viscosity that increases in radius as $\sqrt{r}$ (model $\mathrm{f}$ of Table 1 ). The bold solid line corresponds to a disk whose inner boundary lies on a star whose radius is very small compared to the orbital radius of the planet $\left(r_{*}=0\right)$. The faint solid line is for an inner boundary $r_{*}=0.4 r_{p}$, typically applied in multidimensional hydrodynamical simulations. The dashed line is for the same disk without a planet. The unit of density in each plot is the density of the corresponding disk without a planet at $r=r_{p}$.

cluding the gap region. These solutions are combined with numerical simulations that include the gap region to provide approximate steady state global models.

There is evidence in favor of the models being a reasonable representation of a steady state configuration. In particular, there is little indication of significant changes to the density profiles over the course of the numerical simulations (see Figure 2). The density values at the inner boundary in the numerical simulations (which are free to change) change little. The steady state semi-analytic model makes a parameter-free prediction for the ratio of accretion onto the planet to the rate of accretion past the planet. The numerical simulations are in reasonable agreement with the predicted steady state ratio, generally within $25 \%$. The effects of changing the radius of the star on the accretion rate obtained by the numerical models are also in accord with the predictions of the steady state analytic model.

For a disk with thickness ratio $H / r=0.05$ and planet to star mass ratios $q$ of order $1 \times 10^{-3}$ or less, about $10 \%$ or more of the accretion flow continues past the orbit of the planet and onto the central star. Such reductions would probably still typically produce detectable accretion onto the central star. Most of the accretion matter flows onto the planet. As mentioned earlier, we have generally assumed that the planet can accrete the material that reaches it, in accord with the expectations of current planet formation theory by Pollack et al. (1996) for the mass range we consider. If that assumption were incorrect to the extent that the planet does not accrete any mass, then the star should accrete at the rate expected in the absence of a $q \lesssim 1 \times 10^{-3}$ planet.

The effect of an accreting planet is to decrease the disk density over several times the planet's orbital radius (see Figure 3). The presence of an accreting planet can change the large-scale disk density distribution from a simple power law in radius. Inner circumstellar disks (interior to the orbit of the planet) occur in a steady state, even in the presence of a Jupiter-mass planet. The disk density inside the orbit of the planet (but outside the gap) is decreased as much as about an order of magnitude, due to the presence of a planet. Such inner disks may be missed or underestimated in numerical simulations whose inner boundaries are not sufficiently close to the star (see Figure 3).

Producing cleaner inner holes with lower accretion rates onto the central star may be possible with mass ratios in excess of $q=1 \times 10^{-3}$. For planet to star mass ratios $q \sim$ $5 \times 10^{-3}$, clean inner holes could result (Lubow, Seibert, \& Artymowicz 1999). But, accretion may still occur at higher planet masses, if the disk becomes eccentric as a consequence of a tidal instability (Papaloizou, Nelson, \& Masset 2001; Kley \& Dirksen 2005; D'Angelo, Lubow, \& Bate 2005).

For fixed disk viscosity and planet mass, a colder disk has less ability to penetrate the gap around a planet. The results (model h of Table 1) show that some reduction in flow through the gap occurs with a colder disk. For a mass ratio $q=$ $1 \times 10^{-3}$, the flow is reduced by about $25 \%$ when $H / r$ is reduced from 0.05 to 0.03 .

In the case of the TW Hya, on the basis of low near-IR excess, there is evidence of dust depletion inside $4 \mathrm{AU}$, although the depletion is not complete (Calvet et al. 2002). Based on emission, there is evidence of gas accretion that is one or two orders of magnitude lower than typical for much younger T Tauri stars (Muzerolle et al. 2000) . Some of the reduction could be due to overall gas depletion associated with its $\sim 10$ Myr age due to accretion. It is also possible that the presence of a planet of one Jupiter mass or less mass could play a role in decreasing, but not terminating, the accretion 
in the inner region, by an order of magnitude. Similar considerations apply to the case of $\mathrm{CoKu} \mathrm{Tau} / 4$, where there is evidence for dust depletion inside of $10 \mathrm{AU}$ and no evidence of accretion (D'Alessio et al. 2005). We do not find that inner disk accretion can be suppressed by several orders of magnitude for the planet mass range considered $\left(\lesssim 1 M_{J}\right)$. Such suppression may be possible with a planet of several $M_{J}$. But as mentioned above, even this possibility is uncertain and requires further investigation.

\section{Acknowledgments}

We thank Jim Pringle for helpful discussions. We also thank the referee for useful comments that led to an improvement of the paper. The computations reported in this paper were performed using the UK Astrophysical Fluids Facility (UKAFF). GD is a UKAFF Fellow and acknowledges support from the STScI Visitors Program. SHL acknowledges support from NASA Origins of Solar Systems grant NNG04GG50G.

\section{REFERENCES}

Artymowicz, P., \& Lubow, S. H. 1996, ApJ, 467, L77

Bate, M. R., Ogilvie, G. I., Lubow, S. H., \& Pringle, J. E. 2002, MNRAS, 332, 575

Bate, M. R., Lubow, S. H., Ogilvie, G. I., \& Miller, K. A. 2003, MNRAS, 341, 213

Bryden, G., Chen, X., Lin, D. N. C., Nelson, R. P., \& Papaloizou, J. C. B. 1999, ApJ, 514, 344

Calvet, N., D'Alessio, P., Hartmann, L., Wilner, D., Walsh, A., \& Sitko, M. 2002, ApJ, 568, 1008

Clampin, M., et al. 2003, AJ, 126, 385

D'Alessio, P. et al. 2005 ApJ, 621, 461

D’Angelo, G., Henning, T., \& Kley, W. 2002, A\&A, 385, 647

D’Angelo, G., Kley, W., \& Henning, T. 2003, ApJ, 586, 540

D’Angelo, G., Lubow, S., \& Bate, M. 2005, American Astronomical Society, DPS meeting \#37, \#31.16

Godon, P. 1996, MNRAS, 282, 1107

Godon, P. 1997, ApJ, 483, 882

Herbst, W., et al. 2002, PASP, 114, 1167

Kley, W. 1999, MNRAS, 303, 696

Kley, W., \& Dirksen, G. 2005, preprint

Lin, D. N. C. \& Papaloizou, J. C. B. 1986, ApJ, 309, 846

Lin, D. N. C. \& Papaloizou, J. C. B. 1993, in Protostars and Planets III, ed. E.H. Levy and M.S. Matthews (Tucson: Univ. Arizona Press), p.749

Lubow, S. H., Seibert, M., \& Artymowicz, P. 1999, ApJ, 526, 1001

Lynden-Bell, D. \& Pringle, J.E. 1974, MNRAS, 168, 603

Muzerolle, J., Calvet, N., Briceño, C., Hartmann, L., \& Hillenbrand, L. 2000, ApJ, 535, L47
Nelson, R. P., \& Papaloizou, J. C. B. 2003, MNRAS, 339, 993

Papaloizou, J. C. B., Nelson, R. P., \& Masset, F. 2001, A\&A, 366,263

Pollack, J. B., Hubickyj, O., Bodenheimer, P., Lissauer, J. J., Podolak, M., \& Greenzweig, Y. 1996, Icarus, 124, 62

Pringle, J.E. 1981, ARA\&A, 19, 137

Pringle, J. E., Verbunt, F., \& Wade, R. A. 1986, MNRAS, 221, 169

Pringle, J. E. 1991, MNRAS, 248, 754

Quillen, A. C., Blackman, E. G., Frank, A., \& Varnière, P. 2004, ApJ, 612, L137

Schneider, G., et al. 1999, ApJ, 513, L127

Takeuchi, T., \& Artymowicz, P. 2001, ApJ, 557, 990

Winters, W. F., Balbus, S. A., \& Hawley, J. F. 2003, ApJ, 589,543

This 2-column preprint was prepared with the AAS LATEX macros v5.0, modified by Gennaro D'Angelo. 